BARI-TH 442/02

FIRENZE DFF-390/06/02

UGVA-DPT-2002 07/1101

\title{
Phonons and gluons in the crystalline color superconducting phase of QCD
}

\author{
R. Casalbuoni ${ }^{a, b}$, E.Fabiano $^{c, d}$, R. Gatto ${ }^{e}$, M. Mannarelli ${ }^{c, d}$ and \\ G. Nardulli ${ }^{c, d}$ \\ ${ }^{a}$ Dipartimento di Fisica, Università di Firenze, I-50125 Firenze, Italia \\ ${ }^{b}$ I.N.F.N., Sezione di Firenze, I-50125 Firenze, Italia \\ ${ }^{c}$ Dipartimento di Fisica, Università di Bari, I-70124 Bari, Italia \\ ${ }^{d}$ I.N.F.N., Sezione di Bari, I-70124 Bari, Italia \\ ${ }^{e}$ Départ. de Physique Théorique, Université de Genève, CH-1211 Genève 4, \\ Suisse
}

\begin{abstract}
The High Density Effective Theory formalism is used to calculate the low energy properties of the phonons and gluons in the LarkinOvchinnikov-Fulde-Ferrell (LOFF) phase of two-flavor QCD. The effective phonon Lagrangian for the cubic crystal structure, which is favored according to a recent study, depends, at the second order in the derivatives, on three parameters which we calculate in this paper. We also compute for soft momenta the effective lagrangian for the gluons of the unbroken $S U(2)_{c}$ group, both for a single plane wave structure and for the cube. In both cases the Meissner gluon mass vanishes as in the case of complete isotropy; on the other hand there is a partial Debye screening due to the existence of blocking regions on the Fermi spheres. For the single plane wave structure the gluon dielectric tensor is non isotropic, while it is isotropic for the cubic crystal, in spite of the intrinsic anisotropy of the structure.
\end{abstract}

\section{Introduction}

The study of the color superconducting phases of QCD [1] [2] has recently received much attention and is at the moment one of the most studied frontier 
problem of QCD [3]. Cold QCD with three degenerate flavors, for very large values of the quark number chemical potential $\mu$, is in the so-called colorflavor locked (CFL) phase [4]. In this phase, pairs of quarks of opposite momenta, each of them lying near the Fermi surface, condense according to the BCS pairing scheme known from ordinary superconductivity.

The CFL phase is expected to persist also below asymptotic values of $\mu$, when the flavor degeneracy is broken, with the strange quark having a larger mass than the lighter quarks. In absence of electrons, the CFL phase is electrically neutral. It has the same number of $u, d$, and $s$ quarks, which corresponds to the lowest energy for the Cooper pairs.

When the value of $\mu$ is further decreased, one expects that pairing between quarks of non vanishing total momentum becomes possible. Each quark momentum will lie near its Fermi surface (Fermi spheres will now be of different radii) [5, 6, 7, 8, 9]. Closeness to their Fermi surfaces for both quarks in the pair will correspond to a lower free energy. Such a possibility for the quark pairing leads to the suggestion of a crystalline color superconductivity [5, 6, 7, 8, 9]. We assume the existence of this phase and we will limit our analysis, as in the existing literature, to two flavors, with $\delta \mu \neq 0$ ( $\delta \mu$ the difference in the quark chemical potentials).

In certain condensed matter systems (ferromagnetic systems with paramagnetic impurities) [10 the possibility of fermion pairing of non vanishing total momentum had already been discussed. The name LOFF phase is therefore commonly used, formed from the initials of the authors of ref. [10].

Translational and rotational invariance are spontaneously broken within a LOFF phase. The gaps are expected to vary according to some crystalline structure. The determination of such a structure is obviously of the uppermost importance and this problem is discussed and approximately solved by Bowers and Rajagopal [11] in a recent paper. To understand the crystalline superconductivity one has indeed to know the space dependence of the Cooper pair condensate. A simple plane wave ansatz had been used for a preliminary understanding of the possible dynamics. The plane wave has the finite momentum of the Cooper pairs, on the assumption that all the possible Cooper pair momenta choose a unique direction. These studies (see [5] and [10]) already lead to the conclusion that LOFF pairing is favored, over BCS pairing or absence of pairing, within a certain window in $\delta \mu$. The window starts with a first order phase transition to the crystalline phase. It ends with a second order transition where translation invariance is restored. The window for QCD with two flavors had been found in ref. [5] by modeling 
quark interactions via four-linear Fermi couplings among quarks and for the case of a plane-wave condensate structure. A much wider window is found however in a model based on gluon exchange [8]. This follows essentially from the fact that gluon exchange favors forward scattering, rather than the s-wave scattering typical of the Fermi couplings. The effective low energy phonon lagrangian for the case of the single plane wave structure has been discussed in [7]. Since translation invariance is only partially broken one phonon is present.

A comprehensive study of the different possible crystal structures for the crystalline color superconducting phase of QCD is presented in [11] where the authors perform a Ginzburg-Landau expansion in the vicinity of the phase transition. Instead of the single plane wave ansatz they now introduce a multiple plane-wave ansatz and find that a face-centered cubic crystal is favored. This structure is built up by eight plane waves directed to the corners of a cube. At least qualitatively it seems true that the cubic crystal remains the favored structure also in complete QCD (although not perhaps at very asymptotic densities). It is important to note that for the cubic crystal structure three phonons should be present since translational invariance is completely broken.

The crystalline color superconductivity has a relevant place in the QCD phase diagram and as such it deserves to be studied and to be known in its main features. Moreover it can play a role in the physics of compact stellar systems where, within the core, a crystalline phase might be reached. The authors of ref. [5] had already pointed out that, if the compact star is rotating, the ensuing rotational vortices may become pinned and as such play a role in the formation of glitches. This subject is certainly interesting, but we shall not discuss it here (for a review see ref. [12]).

We have already mentioned that crystalline superconductivity had initially been proposed in condensed matter physics. The QCD studies, in particular also the present one, may be translated into corresponding properties for condensed systems, at least for the phonon properties. We mention in particular the field of atomic systems, gases of fermionic atoms below their Fermi temperature [13], [14]. An advantage of testing the ideas of crystalline superconductivity on these systems is that their parameter space is less rigid than for QCD. For example see ref. [15].

The plan of the present work is as follows. In section 2 we first briefly review the formalism we will use in this paper, i.e. the High Density Effective Theory (HDET). This effective theory is based on the limit $\mu \rightarrow \infty$ and uses 
velocity dependent fields in the same spirit of the Heavy Quark Effective Theory (HQET) [16] where the limit $m_{Q} \rightarrow \infty$ is taken. It is discussed in [17, 18, 19] and more extensively in ref. [20], where the method and the notations are explained in more detail. In section 3 we discuss the low energy dynamics of the phonons. In section 4 we calculate the parameters of the effective phonon lagrangian. In section 5 we study the properties of the gluons of the unbroken $S U(2)_{c}$ for the case of the plane wave structure and in section 6 we perform the same calculation for the LOFF condensate with the cubic symmetry. The application to the cubic crystal structure requires some group theoretical definitions which are summarized in Appendix 1. In Appendix 2 and 3 we report some details of the calculations.

\section{Heavy Density Effective Theory in the LOFF phase}

We begin with the effective lagrangian of HDET for gluons and ungapped massless quarks. If $\mathcal{L}_{0}$ is the free quark lagrangian and $\mathcal{L}_{1}$ represents the coupling of quarks to one gluon, the high density effective lagrangian can be written as

$$
\mathcal{L}_{D}=\mathcal{L}_{0}+\mathcal{L}_{1}+\mathcal{L}_{2}+(L \rightarrow R) .
$$

Let us begin with the first two terms, i.e.

$$
\begin{aligned}
\mathcal{L}_{0} & =\sum_{\vec{v}}\left[\psi_{+}^{\dagger} i V \cdot \partial \psi_{+}+\psi_{-}^{\dagger} i \tilde{V} \cdot \partial \psi_{-}\right] \\
\mathcal{L}_{1} & =i g \sum_{\vec{v}}\left[\psi_{+}^{\dagger} i V \cdot A \psi_{+}+\psi_{-}^{\dagger} i \tilde{V} \cdot A \psi_{-}\right] .
\end{aligned}
$$

The velocity dependent left-handed field $\psi_{+} \equiv \psi_{\vec{v}}$ is the positive energy part in the decomposition

$$
\psi(x)=\int \frac{d \vec{v}}{4 \pi} e^{-i \mu v \cdot x}\left[\psi_{\vec{v}}(x)+\Psi_{\vec{v}}(x)\right]
$$

while $\Psi_{\vec{v}}$ is the negative energy part which has been integrated out. Here

$$
\psi_{\vec{v}}(x)=e^{i \mu v \cdot x} P_{+} \psi(x)=\int_{|\ell|<\delta} \frac{d^{4} \ell}{(2 \pi)^{4}} e^{-i \ell \cdot x} P_{+} \psi(\ell)
$$


and

$$
\Psi_{\vec{v}}(x)=e^{i \mu v \cdot x} P_{-} \psi(x)=\int_{|\ell|<\delta} \frac{d^{4} \ell}{(2 \pi)^{4}} e^{-i \ell \cdot x} P_{-} \psi(\ell) .
$$

$P_{ \pm}$are projectors defined by

$$
P_{ \pm}=\frac{1}{2}(1 \pm \vec{\alpha} \cdot \vec{v})
$$

The cut-off $\delta$ satisfies $\delta \ll \mu$ while being much larger that the energy gap. These equations correspond to the following decomposition of the quark momentum:

$$
p^{\mu}=\mu v^{\mu}+\ell^{\mu}
$$

where

$$
v^{\mu}=(0, \vec{v})
$$

and $|\vec{v}|=1$. We also use $V^{\mu}=(1, \vec{v}), \tilde{V}^{\mu}=(1,-\vec{v})$.

The other velocity-dependent field $\psi_{-} \equiv \psi_{-\vec{v}}$ is obtained from $\psi_{+}$by the change $\vec{v} \rightarrow-\vec{v}$. Therefore the average over the Fermi velocities is defined as follows:

$$
\sum_{\vec{v}}=\int \frac{d \vec{v}}{8 \pi}
$$

The extra factor $\frac{1}{2}$ is introduced because, after the introduction of the field with opposite velocity $\psi_{-}$, one doubles the degrees of freedom, which implies that the integration is only over half solid angle.

The effective lagrangian (11) is obtained in the limit $\mu \rightarrow \infty$ 17, 18, 19, 20]. Therefore $\mathcal{L}_{0}$ and $\mathcal{L}_{1}$ do not contain the chemical potential because the dependence on $\mu$ has been extracted from the quark field operators. We also note that one has a single sum over the velocities, because the quark fields are evaluated at the same Fermi velocity; as a matter of fact the off-diagonal terms are cancelled by the rapid oscillations of the exponential factor in the $\mu \rightarrow \infty$ limit. This point will be discussed in a more detailed way in the sequel.

Besides $\mathcal{L}_{0}$ and $\mathcal{L}_{1}$ eq.(II) contains the term

$$
\mathcal{L}_{2}=-\sum_{\vec{v}} P^{\mu \nu}\left[\psi_{+}^{\dagger} \frac{1}{2 \mu+i \tilde{V} \cdot D} D_{\mu} D_{\nu} \psi_{+}+\psi_{-}^{\dagger} \frac{1}{2 \mu+i V \cdot D} D_{\mu} D_{\nu} \psi_{-}\right] .
$$

$\mathcal{L}_{2}$ is a non local lagrangian arising when one integrates over the $\Psi_{\vec{v}}$ degrees of freedom in the functional integration. It contains couplings of two quarks 
to any number of gluons and gives contribution to the gluon Meissner mass (see below). We have put

$$
P^{\mu \nu}=g^{\mu \nu}-\frac{1}{2}\left[V^{\mu} \tilde{V}^{\nu}+V^{\nu} \tilde{V}^{\mu}\right] .
$$

This construction is valid for any theory describing massless fermions at high density provided one excludes degrees of freedom far from the Fermi surface. Let us now examine the modification in the formalism in the presence of a LOFF condensate; to begin with we consider the approximation of one plane wave. The space dependence of the crystalline condensate in this case is

$$
\Delta(\vec{x})=\Delta \exp \{2 i \vec{q} \cdot \vec{x}\}
$$

The effect of the non vanishing vacuum expectation value can be taken into account by adding to the lagrangian the term:

$$
\mathcal{L}_{\Delta}=-\frac{\Delta}{2} \exp \{2 i \vec{q} \cdot \vec{x}\} \epsilon_{\alpha \beta 3} \epsilon_{i j} \psi_{i \alpha}^{T}(x) C \psi_{j \beta}(x)-(L \rightarrow R)+\text { h.c. . }
$$

In order to introduce velocity dependent positive energy fields $\psi_{\vec{v}_{i} ; i \alpha}$ with flavor $i$ we decompose both fermion momenta as in (8) and we have:

$$
\begin{aligned}
\mathcal{L}_{\Delta}= & -\frac{\Delta}{2} \sum_{\vec{v}_{i}, \vec{v}_{j}} \exp \left\{i \vec{x} \cdot \vec{\alpha}\left(\vec{v}_{i}, \vec{v}_{j}, \vec{q}\right)\right\} \epsilon_{i j} \epsilon_{\alpha \beta 3} \psi_{-\vec{v}_{i} ; i \alpha}^{T}(x) C \psi_{-\vec{v}_{j} ; j \beta}(x) \\
& -(L \rightarrow R)+\text { h.c. }
\end{aligned}
$$

where

$$
\vec{\alpha}\left(\vec{v}_{i}, \vec{v}_{j}, \vec{q}\right)=2 \vec{q}-\mu_{i} \vec{v}_{i}-\mu_{j} \vec{v}_{j}
$$

Let us define

$$
\mu=\frac{\mu_{1}+\mu_{2}}{2}, \quad \delta \mu=-\frac{\mu_{1}-\mu_{2}}{2} .
$$

We will consider large values of $\mu$ (with $\delta \mu / \mu \rightarrow 0, q / \delta \mu \rightarrow 0$ and $\delta \mu / q$ fixed), but below the value where the CFL phase sets in. To give our procedure a mathematical meaning, we consider a smeared amplitude as follows:

$$
\lim _{\mu \rightarrow \infty} \exp \left\{i \vec{r} \cdot \vec{\alpha}\left(\vec{v}_{1}, \vec{v}_{2}, \vec{q}\right)\right\} \equiv \lim _{\mu \rightarrow \infty} \int d \vec{r}^{\prime} \exp \left\{i \vec{r}^{\prime} \cdot \vec{\alpha}\left(\vec{v}_{1}, \vec{v}_{2}, \vec{q}\right)\right\} g\left(\vec{r}, \vec{r}^{\prime}\right)
$$

In [21] we assumed the following smearing function:

$$
g\left(\vec{r}, \vec{r}^{\prime}\right)=g_{I}\left(\vec{r}-\vec{r}^{\prime}\right)=\prod_{k=1}^{3} \theta_{\Delta \ell}\left(r_{k}-r_{k}^{\prime}\right)
$$


where

$$
\begin{aligned}
\theta_{\Delta \ell}\left(r-r^{\prime}\right) & =\frac{1}{\Delta \ell}\left[\theta\left(r^{\prime}-r+\frac{\Delta \ell}{2}\right)-\theta\left(r^{\prime}-r-\frac{\Delta \ell}{2}\right)\right]= \\
& =\left\{\begin{array}{cc}
\frac{1}{\Delta \ell} & \text { for }\left|r^{\prime}-r\right|<\frac{\Delta \ell}{2} \\
0 & \text { elsewhere }
\end{array}\right.
\end{aligned}
$$

( $\theta$ the Heaviside function). In the present paper, for reasons to be discussed below, we will choose

$$
g\left(\vec{r}, \vec{r}^{\prime}\right)=g_{I I}\left(\vec{r}-\vec{r}^{\prime}\right)=\prod_{k=1}^{3} \frac{\sin \left[\frac{\pi q\left(r_{k}-r_{k}^{\prime}\right)}{R}\right]}{\pi\left(r_{k}-r_{k}^{\prime}\right)} .
$$

We evaluate (18) in the $\mu \rightarrow \infty$ limit by taking $\vec{q}$ along the $z$-axis, and using the following identity:

$$
\int d^{3} \vec{r}^{\prime} \exp \left\{i \vec{r}^{\prime} \cdot \vec{\alpha}\right\} g_{I I}\left(\vec{r}-\vec{r}^{\prime}\right)=\exp \{i \vec{r} \cdot \vec{\alpha}\}\left(\frac{\pi}{R}\right)^{3} \delta_{R}^{3}\left(\frac{\vec{\alpha}}{2 q}\right)
$$

where

$$
\delta_{R}(x)=\left\{\begin{array}{cc}
\frac{R}{\pi} & \text { for }|x|<\frac{\pi}{2 R} \\
0 & \text { elsewhere }
\end{array}\right.
$$

For the components $x$ and $y$ of $\vec{\alpha}$ we get

$$
\left|\left(\mu_{1} v_{1}+\mu_{2} v_{2}\right)_{x, y}\right|<\frac{\pi q}{R}
$$

i.e. approximately (for $\delta \mu \ll \mu$ )

$$
\left|\left(v_{1}+v_{2}\right)_{x, y}\right|<\frac{\pi q}{R \mu} .
$$

From this in the high density limit it follows

$$
\vec{v}_{1}=-\vec{v}_{2}+\mathcal{O}(1 / \mu)
$$


More precisely, if $\theta_{1}$ and $\theta_{2}$ are the angles of $\vec{v}_{1}$ and $\vec{v}_{2}$ with respect to the $z$-axis one gets

$$
\theta_{1}=\theta_{2}+\pi+2 \frac{\delta \mu}{\mu} \tan \theta_{2}
$$

For the $z$ component we get

$$
\alpha_{z}=2 q h\left(\cos \theta_{2}\right)
$$

The expression for $h(x)$ is [21]:

$$
h(x)=1+\frac{x \mu_{2}}{2 q}\left(-1+\sqrt{1-\frac{4 \mu \delta \mu}{\mu_{2}^{2} x^{2}}}\right) .
$$

Neglecting corrections of order $\delta \mu / \mu$ one finds

$$
h(x)=1-\frac{\cos \theta_{q}}{x}, \quad \cos \theta_{q}=\frac{\delta \mu}{q} .
$$

The two factors $\pi / R$ arising from the $x$ and $y$ components are absorbed into a wave function renormalization of the quark fields, both in the kinetic and in the gap terms. As for the $z$ component one remains with the factor

$$
\frac{\pi}{R} e^{i 2 q h z} \delta_{R}[h(\vec{v} \cdot \vec{n})] \approx \frac{\pi}{R} \delta_{R}[h(\vec{v} \cdot \vec{n})]
$$

where

$$
\vec{n}=\vec{q} / q
$$

in the gap term, whereas for the kinetic term we get a factor of 1 .

As we noted already, in ref. [21] we used as a smearing function $g_{I}$ (see eq. (19)) and $\delta_{R}$ turned out to be given by

$$
\delta_{\hat{R}}(x)=\frac{\sin (\hat{R} x)}{\pi x}, \quad \hat{R}=q|\Delta \ell| .
$$

Both eqns. (33) and (23) define regions where $\delta_{R} \neq 0$, i.e. define domains where pairing between the two quarks can occur; they correspond to the pairing region (in contrast with the blocking region, where $\delta_{R}=0$ ), using the terminology of [4]. However, given the results of [11], which show that the different sections of the pairing domain for the LOFF cubic structure are nonoverlapping, we prefer to choose the prescription (21) and, consequently, eq. 
(23) for $\delta_{R}$. We explicitly observe that, owing to (30), with $x=\vec{v} \cdot \vec{n}=\cos \theta$, eq. (23) defines a 'ring' on the Fermi sphere. Our approximation consists in putting $\exp [i 2 q h z]=1$ in eq. (31), owing to the presence of $\delta_{R}$ that enhances the domain of integration where $h=0$, for $R$ sufficiently large.

Let us discuss the range of values of $R$ 回 for the plane wave ansatz in more detail. We notice that $R=\infty$ implies the vanishing of the pairing region. The precise value of $R$ could be fixed by the gap equation. For instance, by using the fact that near the second order phase transition the pairing region has an angular width of order $\Delta / \delta \mu$ (see for a discussion [11]), from the definition of $\delta_{R}$ we find (for $\Delta \rightarrow 0$ )

$$
\frac{\pi}{R} \approx \frac{\Delta}{\delta \mu} \sqrt{\left(\frac{q}{\delta \mu}\right)^{2}-1} .
$$

This shows that $R \rightarrow \infty$ for $\Delta \rightarrow 0$. In any case for the purpose of this paper we leave $R$ as a parameter.

In conclusion

$$
\mathcal{L}_{\Delta}=-\frac{\Delta}{2} \frac{\pi}{R} \sum_{\vec{v}} \delta_{R}[h(\vec{v} \cdot \vec{n})] \epsilon_{i j} \epsilon_{\alpha \beta 3} \psi_{\vec{v} ; i \alpha}^{T}(x) C \psi_{-\vec{v} ; j \beta}(x)-(L \rightarrow R)+\text { h.c. }
$$

We now change the basis for the fermion fields by writing

$$
\begin{aligned}
\psi_{+, \alpha i} & =\sum_{A=0}^{3} \frac{\left(\sigma_{A}\right)_{\alpha i}}{\sqrt{2}} \psi_{+}^{A} \quad(i, \alpha=1,2) \\
\psi_{+, 31} & =\psi_{+}^{4} \\
\psi_{+, 32} & =\psi_{+}^{5}
\end{aligned}
$$

where $\sigma_{A}$ are the Pauli matrices for $A=1,2,3$ and $\sigma_{0}=1$; similar expressions hold for $\psi_{-}^{A}$.

A different, but also useful notation for the fields $\psi_{+, \alpha i}$ uses a combination of $\lambda$ matrices, as follows

$$
\psi_{+, \alpha i}=\sum_{A=0}^{5} \frac{\left(\tilde{\lambda}_{A}\right)_{\alpha i}}{\sqrt{2}} \psi_{+}^{A} .
$$

The $\tilde{\lambda}_{A}$ matrices are defined in terms of the usual $\lambda$ matrices as follows: $\tilde{\lambda}_{0}=\frac{1}{\sqrt{3}} \lambda_{8}+\sqrt{\frac{2}{3}} \lambda_{0} ; \tilde{\lambda}_{A}=\lambda_{A}(A=1,2,3) ; \tilde{\lambda}_{4}=\frac{\lambda_{4-i 5}}{\sqrt{2}} ; \tilde{\lambda}_{5}=\frac{\lambda_{6-i 7}}{\sqrt{2}}$. In this

\footnotetext{
${ }^{1}$ Notice that the quantity $R$ used in the present work is not the same used in 21$]$.
} 
basis one gets

$$
\begin{aligned}
\mathcal{L}_{0}+\mathcal{L}_{1}+\mathcal{L}_{\Delta} & =\sum_{\vec{v}} \sum_{A, B=0}^{5} \chi^{A \dagger}\left(\begin{array}{cc}
i \operatorname{Tr}\left[\tilde{T}_{A}^{\dagger} V \cdot D \tilde{T}_{B}\right] & \Delta_{A B} \\
\Delta_{A B} & i \operatorname{Tr}\left[\tilde{T}_{A}^{\dagger} \tilde{V} \cdot D^{*} \tilde{T}_{B}\right]
\end{array}\right) \chi^{B} \\
& +(L \rightarrow R) .
\end{aligned}
$$

Here

$$
\chi^{A}=\frac{1}{\sqrt{2}}\left(\begin{array}{c}
\psi_{+}^{A} \\
C \psi_{-}^{A *}
\end{array}\right)
$$

and

$$
\tilde{T}_{A}=\frac{\tilde{\lambda}_{A}}{\sqrt{2}} \quad(A=0, \ldots, 5) .
$$

where the matrix $\Delta_{A B}$ is as follows: $\Delta_{A B}=0(A$ or $B=4$ or 5$)$, and, for $A, B=0, \ldots, 3$ :

$$
\Delta_{A B}=\Delta_{e f f}\left(\begin{array}{cccc}
1 & 0 & 0 & 0 \\
0 & -1 & 0 & 0 \\
0 & 0 & -1 & 0 \\
0 & 0 & 0 & -1
\end{array}\right)_{A B}
$$

having put

$$
\Delta_{e f f}=\frac{\Delta \pi}{R} \delta_{R}[h(\vec{v} \cdot \vec{n})] .
$$

From these equations one can derive the propagator for gapped quarks:

$$
D_{A B}(\ell)=\frac{1}{V \cdot \ell \tilde{V} \cdot \ell-\Delta_{e f f}^{2}}\left(\begin{array}{cc}
\tilde{V} \cdot \ell \delta_{A B} & -\Delta_{A B} \\
-\Delta_{A B} & V \cdot \ell \delta_{A B}
\end{array}\right) .
$$

On the other hand the propagator for the fields $\chi^{4,5}$ does not contain gap mass terms and is given by

$$
D(\ell)=\left(\begin{array}{cc}
(V \cdot \ell)^{-1} & 0 \\
0 & (\tilde{V} \cdot \ell)^{-1}
\end{array}\right) .
$$

These formulae can be easily generalized to the case of the face centered cube [11]. This is characterized by eight $\vec{q}_{j}$ vectors with equal length $q=\pi / a$ and directions corresponding to the eight vertices of a cube.

In Fig. 1 some of the symmetry axes of this cube are shown: they are denoted as $C_{4}$ (the three 4 -fold axes), $C_{3}$ (the four 3 -fold axes) and $C_{2}$ (the 
six 2-fold axes). For more details on the symmetry group of the cube see Appendix 1 and [22]. The space dependence of the condensate corresponding to this lattice is as follows

$$
\Delta(\vec{x})=\Delta \sum_{k=1}^{8} \exp \left\{2 i \vec{q}_{k} \cdot \vec{x}\right\}=\Delta \sum_{\epsilon_{i}= \pm} \exp \left\{\frac{2 \pi i}{a}\left(\epsilon_{1} x_{1}+\epsilon_{2} x_{2}+\epsilon_{3} x_{3}\right)\right\},
$$

where the eight $\vec{q}_{j}$ vectors with equal length

$$
q=\pi / a
$$

have directions corresponding to the eight vertices of a cube.

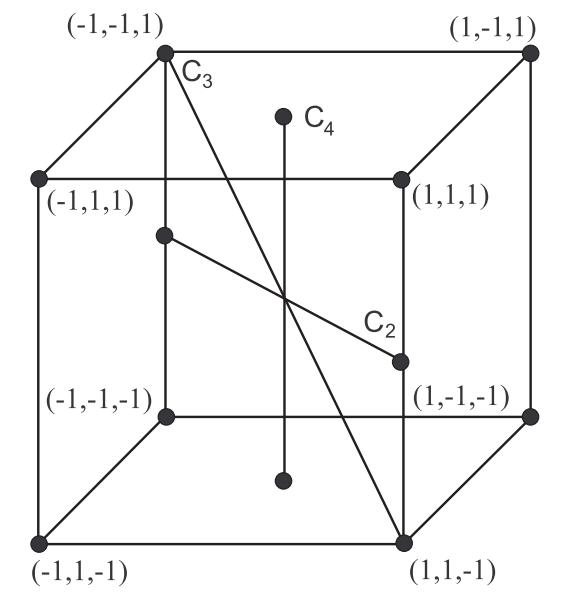

Fig. 1 - The figure shows some of the symmetry axes of the cube, denoted as $C_{2}, C_{3}$ and $C_{4}$ (see the appendix for details). We show also the parameterization used for the coordinates of the vertices.

To describe the quark condensate we add the term:

$$
\mathcal{L}_{\Delta}=-\frac{\Delta}{2} \sum_{k=1}^{8} \exp \left\{2 i \vec{q}_{k} \cdot \vec{x}\right\} \epsilon_{\alpha \beta 3} \epsilon_{i j} \psi_{i \alpha}^{T}(x) C \psi_{j \beta}(x)-(L \rightarrow R)+\text { h.c. }
$$

completely analogous to (14).

Let us put

$$
\vec{n}_{k}=\vec{q}_{k} /\left|\vec{q}_{k}\right|
$$


By the same procedure used for the plane wave condensate one has

$$
\begin{aligned}
\mathcal{L}_{\Delta}= & -\frac{\Delta}{2} \sum_{k=1}^{8} \sum_{\vec{v}} \frac{\pi}{R} \delta_{R}\left[h\left(\vec{v} \cdot \vec{n}_{k}\right)\right] \epsilon_{i j} \epsilon_{\alpha \beta 3} \psi_{\overrightarrow{;} ; i \alpha}^{T}(x) C \psi_{-\vec{v} ; j \beta}(x) \\
& -(L \stackrel{R}{\rightarrow})+\text { h.c. }
\end{aligned}
$$

In (48) the eight unit vectors defining the vertices of the cube are

$$
\begin{array}{ll}
\vec{n}_{1}=\frac{1}{\sqrt{3}}(+1,+1,+1), & \vec{n}_{2}=\frac{1}{\sqrt{3}}(+1,-1,+1), \\
\vec{n}_{3}=\frac{1}{\sqrt{3}}(-1,-1,+1), & \vec{n}_{4}=\frac{1}{\sqrt{3}}(-1,+1,+1), \\
\vec{n}_{5}=\frac{1}{\sqrt{3}}(+1+, 1,-1), & \vec{n}_{6}=\frac{1}{\sqrt{3}}(+1,-1,-1), \\
\vec{n}_{7}=\frac{1}{\sqrt{3}}(-1,-1,-1), & \vec{n}_{8}=\frac{1}{\sqrt{3}}(-1,+1,-1) .
\end{array}
$$

$\mathcal{L}_{0}+\mathcal{L}_{1}+\mathcal{L}_{\Delta}$ is still given by eqns. (38)-(41) but now

$$
\Delta_{e f f}=\frac{\Delta \pi}{R} \sum_{k=1}^{8} \delta_{R}\left[h\left(\vec{v} \cdot \vec{n}_{k}\right)\right] ;
$$

the quark propagator is given by (43) with $\Delta_{\text {eff }}$ given by (51).

An interesting point can be noted in connection with eq. (51). This equation shows that the pairing region for the cubic LOFF condensate is formed by eight distinct rings; each ring is associated to one vertex of the cube and has as its symmetry axis one of the $C_{3}$ axes of fig. 1. According to the analysis of [11] the LOFF vacuum state corresponds to a situation where these domains have at most one common point. Given the symmetry of the cubic structure we can limit the analysis to one pair of rings, for example those associated to the vertices $\vec{n}_{1}, \vec{n}_{5}$ in fig. 1 . The common point between these two rings lies on the axis $C_{2}$ of fig. 1 and has $\vec{v}=\frac{1}{\sqrt{2}}(1,1,0)$. Since it must also belong to the boundary of the two pairing regions we have the condition (23):

$$
\left|h\left(\vec{v} \cdot \vec{n}_{1}\right)\right|=\frac{\pi}{2 R},
$$

which implies

$$
R=\frac{\pi}{2 h(\sqrt{2 / 3})}
$$


In this particular case the approximate form for $h(x)$ (see eq. (30)) is not appropriate owing a numerical cancellation. In fact for $q /|\delta \mu| \approx 1.2, \mu=$ $400 \mathrm{MeV}, \delta \mu=40 \mathrm{MeV}$, one would get $R \approx 76$, whereas the use of the complete equation $(29)$ gives

$$
R \approx 18
$$

\section{Phonons in a cubic LOFF crystal}

The condensate (45) breaks both translations and rotations. It is however invariant under the discrete group $O_{h}$, the symmetry group of the cube. This can be seen by noticing that the condensate is invariant under the following coordinate transformations

$$
\begin{array}{ll}
R_{1}: & x_{1} \rightarrow x_{1}, \quad x_{2} \rightarrow x_{3}, \quad x_{3} \rightarrow-x_{2}, \\
R_{2}: & x_{1} \rightarrow-x_{3}, \quad x_{2} \rightarrow x_{2}, \quad x_{3} \rightarrow x_{1}, \\
R_{3}: & x_{1} \rightarrow x_{2}, \quad x_{2} \rightarrow-x_{1}, \quad x_{3} \rightarrow x_{3}, \\
I: & x_{1} \rightarrow-x_{1}, \quad x_{2} \rightarrow-x_{2}, \quad x_{3} \rightarrow-x_{3},
\end{array}
$$

that is rotations of $\pi / 2$ around the coordinate axes, and the inversion with respect to the origin. Since the group $O_{h}$ is generated by the previous 4 elements (see Appendix 1) the invariance follows at once.

The crystal defined by the condensate (45) can fluctuate and its local deformations define three phonon fields $\phi^{(i)}$ that are the Nambu-Goldstone bosons associated to the breaking of the translational symmetry. They can be formally introduced by the substitution in (45)

$$
2 q x^{i} \rightarrow \Phi^{(i)}(x)=\frac{2 \pi}{a} x^{i}+\phi^{(i)}(x) / f,
$$

where the three scalar fields $\Phi^{(i)}$ satisfy

$$
\left\langle\Phi^{(i)}\right\rangle_{0}=\frac{2 \pi}{a} x_{i}
$$

whereas for the phonon fields one has

$$
\left\langle\phi^{(i)}(x)\right\rangle_{0}=0 \text {. }
$$


In (56) $f$ is the decay constant of the phonon with dimension of an energy. We have therefore three fluctuating fields $\phi_{k_{1} k_{2} k_{3}}^{(i)}$ for any elementary cube defined by discrete coordinates

$$
x_{k_{1}}=\frac{k_{1} \pi}{q}, \quad y_{k_{2}}=\frac{k_{2} \pi}{q}, z_{k_{3}}=\frac{k_{3} \pi}{q},
$$

i.e.

$$
\phi_{k_{1} k_{2} k_{3}}^{(i)} \equiv \phi^{(i)}\left(t, x_{k_{1}}, y_{k_{2}}, z_{k_{3}}\right) .
$$

The interaction term with the NGB fields will be therefore given by

$$
\begin{aligned}
S_{i n t} & =-\int d t\left(\frac{\pi}{q}\right)^{3} \sum_{k_{1}=-\infty}^{+\infty} \sum_{k_{2}=-\infty}^{+\infty} \sum_{k_{3}=-\infty}^{+\infty} \sum_{\vec{v}} \sum_{m=1}^{8} \Delta \exp \left\{i \varphi_{k_{1} k_{2} k_{3}}^{(m)} / f\right\} \\
& \times \epsilon_{i j} \epsilon^{\alpha \beta 3} \psi_{i, \alpha, \vec{v}}^{T} C \psi_{j, \beta,-\vec{v}}-(L \rightarrow R)+\text { h.c. }
\end{aligned}
$$

where

$$
\varphi_{k_{1} k_{2} k_{3}}^{(m)}=\sum_{i=1}^{3} \epsilon_{i}^{(m)} \phi_{k_{1} k_{2} k_{3}}^{(i)}
$$

and the eight vectors $\vec{\epsilon}^{(m)}$ are given by

$$
\left(\epsilon_{i}^{(m)}\right) \equiv \vec{\epsilon}^{(m)} \equiv \sqrt{3} \vec{n}_{m}
$$

Notice that translating the fields in momentum space one would have to consider Fourier series with respect to the discrete coordinates. This introduces in the theory a quasi-momentum $\vec{p}$ through the combination $\exp \left(i\left(p_{x} x_{k_{1}}+\right.\right.$ $\left.p_{y} y_{k_{2}}+p_{z} z_{k_{3}}\right)$. Therefore one has to restrict $\vec{p}$ to the first Brillouin zone $-q \leq p_{x} \leq q,-q \leq p_{y} \leq q,-q \leq p_{z} \leq q$.

The complete effective action for the NGB fields $\phi^{(i)}$ will be of the form

$$
S=\int d t\left(\frac{\pi}{q}\right)^{3} \sum_{k_{1}=-\infty}^{+\infty} \sum_{k_{2}=-\infty}^{+\infty} \sum_{k_{3}=-\infty}^{+\infty} \mathcal{L}\left(\phi^{(i)}\left(t, k_{1} \pi / q, k_{2} \pi / q, k_{3} \pi / q\right)\right)
$$

In the action bilinear terms of the type $\phi_{k_{1} k_{2} k_{3}}^{(i)} \phi_{k_{1}^{\prime} k_{2}^{\prime} k_{3}^{\prime}}^{\left(i^{\prime}\right)}$ with $\left\{k_{1} k_{2} k_{3}\right\} \neq$ $k_{1}^{\prime} k_{2}^{\prime} k_{3}^{\prime}$ may arise. In the continuum limit this terms would correspond to partial derivatives with respect to the three space directions.

All these considerations become superfluous when one realizes that we are actually interested in an effective description of the fields $\phi_{k_{1}, k_{2}, k_{3}}^{(i)}$ in the low energy limit, i.e. for wavelengths much longer than the lattice spacing $\sim 1 / q$. 
In this limit the fields $\phi_{k_{1}, k_{2}, k_{3}}^{(i)}$ vary almost continuously and can be imagined as continuous functions of three space variables $x, y$ and $z$. Therefore we shall use in the sequel the continuous notation $\phi^{(i)}(t, \vec{r})$.

The coupling of the quark fields to the NGB fields generated by the condensate will be written as

$$
\Delta \psi^{T} C \psi \sum_{\epsilon_{i}= \pm} \exp \left\{i\left(\epsilon_{1} \Phi^{(1)}+\epsilon_{2} \Phi^{(2)}+\epsilon_{3} \Phi^{(3)}\right)\right\}
$$

making the theory invariant under translations and rotations. These invariances are broken spontaneously in the vacuum defined by eq. (57).

Integrating out the Fermi degrees of freedom one gets an effective lagrangian containing only the light degrees of freedom, i.e. the gluons of the unbroken subgroup $S U(2)_{c}$ and the phonons. We will discuss gluons in Section 6 and we consider here only the phonon effective lagrangian.

The effective lagrangian for the fields $\Phi^{(i)}$ has to enjoy the following symmetries: rotational and translational invariance; $O_{h}$ symmetry on the fields $\Phi^{(i)}$. The latter requirement follows from the invariance of the coupling (65) under the group $O_{h}$ acting upon $\Phi^{(i)}$. The phonon fields $\phi^{(i)}(x)$ and the coordinates $x^{i}$ must transform under the diagonal discrete group obtained from the direct product of the rotation group acting over the coordinates and the $O_{h}$ group acting over $\Phi^{(i)}(x)$. This is indeed the symmetry left after the breaking of translational and rotational invariance.

In order to build up the effective lagrangian, we start noticing that if $X_{i}$ are quantities transforming as the same representation of the $\Phi^{(i)}$ 's under $O_{h}$ (a 3-dimensional representation), then all the invariant expressions can be obtained by the following three basic invariant expressions

$I_{2}\left(X_{i}\right)=X_{1}^{2}+X_{2}^{2}+X_{3}^{2}, I_{4}\left(X_{i}\right)=X_{1}^{2} X_{2}^{2}+X_{2}^{2} X_{3}^{2}+X_{3}^{2} X_{1}^{2}, \quad I_{6}\left(X_{i}\right)=X_{1}^{2} X_{2}^{2} X_{3}^{2}$,

which is a general property of the symmetric functions of three variables. The invariance is easily checked by noticing that these expressions are invariant under the three elementary rotations $R_{i}$ and the inversion $I$ (see eq. (55)). Quantities transforming as $\Phi^{(i)}$ are

$$
\dot{\Phi}^{(i)} \equiv \frac{d \Phi^{(i)}}{d t}, \quad \vec{\nabla} \Phi^{(i)} .
$$

We proceed now as in ref. [7], by noticing that the most general low-energy effective lagrangian, invariant under rotations, translations and $O_{h}$ is given 


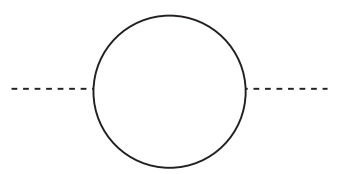

a)

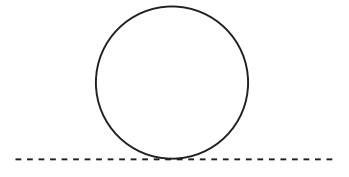

b)

Fig. 2. Self-energy (a) and tadpole (b) diagrams.

by

$$
\mathcal{L}=\frac{f^{2}}{2} \sum_{i=1,2,3}\left(\dot{\Phi}^{(i)}\right)^{2}+\mathcal{L}_{\mathrm{s}}\left(I_{2}\left(\vec{\nabla} \Phi^{(i)}\right), I_{4}\left(\vec{\nabla} \Phi^{(i)}\right), I_{6}\left(\vec{\nabla} \Phi^{(i)}\right)\right) .
$$

The reason for allowing all the possible spatial gradients in this expansion is that the vacuum expectation value of $\vec{\nabla} \Phi^{(i)}$ is proportional to $q$ and it is not in general small. By following ref. [23] one gets the effective lagrangian for the fields $\phi^{(i)}$ at the second order in the derivatives as follows

$\mathcal{L}=\frac{1}{2} \sum_{i=1,2,3}\left(\dot{\phi}^{(i)}\right)^{2}-\frac{a}{2} \sum_{i=1,2,3}\left|\vec{\nabla} \phi^{(i)}\right|^{2}-\frac{b}{2} \sum_{i=1,2,3}\left(\partial_{i} \phi^{(i)}\right)^{2}-c \sum_{i<j=1,2,3} \partial_{i} \phi^{(i)} \partial_{j} \phi^{(j)}$.

The effective theory depends on three arbitrary parameters, a result that follows also from the general theory of the propagation of elastic waves in crystals [24].

\section{Parameters of the effective lagrangian for the phonon fields}

In this Section we will compute the parameters $a, b, c$ appearing in (69). We use the gradient expansion. First we introduce external fields with the same quantum numbers of the NGB's and then we perform a derivative expansion of the generating functional. This gives rise to the effective action for the NGB. At the lowest order one has to consider the diagrams in Fig. 2, i.e. the self-energy, Fig. 2a, and the tadpole, Fig. 2b. The diagrams can be computed observing that, by expanding (65) in the phonon fields, one has 
couplings of two quarks with one and two phonons. Let us put

$$
\varphi^{(m)}(t, \vec{r})=\sum_{i=1}^{3} \epsilon_{i}^{(m)} \phi^{(i)}(t, \vec{r})
$$

At the first order in the fields one gets the coupling with one NGB

$$
\begin{aligned}
\mathcal{L}_{\phi \psi \psi} & =-\sum_{\vec{v}} \sum_{m=1}^{8} \frac{\pi \Delta}{R} \delta_{R}\left[h\left(\vec{v} \cdot \vec{n}_{m}\right)\right] \frac{\left(i \varphi^{(m)}\right)}{f} \epsilon_{i j} \epsilon^{\alpha \beta 3} \psi_{\vec{v} ; i \alpha} C \psi_{-\vec{v} ; j \beta} \\
& -(L \rightarrow R)+h . c .
\end{aligned}
$$

and the coupling of two quarks and two NGB's

$$
\begin{aligned}
\mathcal{L}_{\phi \phi \psi \psi} & =\sum_{\vec{v}} \sum_{m=1}^{8} \frac{\pi \Delta}{R} \delta_{R}\left[h\left(\vec{v} \cdot \vec{n}_{m}\right)\right] \frac{\left(\varphi^{(m)}\right)^{2}}{2 f^{2}} \epsilon_{i j} \epsilon^{\alpha \beta 3} \psi_{\vec{v} ; i \alpha} C \psi_{-\vec{v} ; j \beta} \\
& -(L \rightarrow R)+\text { h.c. }
\end{aligned}
$$

In the basis of the $\chi$ fields:

$$
\mathcal{L}_{3}+\mathcal{L}_{4}=\sum_{\vec{v}} \sum_{A=0}^{3} \tilde{\chi}^{A \dagger}\left(\begin{array}{cc}
0 & g_{3}^{\dagger}+g_{4}^{\dagger} \\
g_{3}+g_{4} & 0
\end{array}\right) \tilde{\chi}^{B}
$$

Here

$$
\begin{aligned}
g_{3} & =\sum_{m=1}^{8} \frac{\pi \Delta}{R} \delta_{R}\left[h\left(\vec{v} \cdot \vec{n}_{m}\right)\right] \frac{i \varphi^{(m)}}{f}\left(\begin{array}{cccc}
1 & 0 & 0 & 0 \\
0 & -1 & 0 & 0 \\
0 & 0 & -1 & 0 \\
0 & 0 & 0 & -1
\end{array}\right)_{A B}, \\
g_{4} & =-\sum_{m=1}^{8} \frac{\pi \Delta}{R} \delta_{R}\left[h\left(\vec{v} \cdot \vec{n}_{m}\right)\right] \frac{\left(\varphi^{(m)}\right)^{2}}{2 f^{2}}\left(\begin{array}{cccc}
1 & 0 & 0 & 0 \\
0 & -1 & 0 & 0 \\
0 & 0 & -1 & 0 \\
0 & 0 & 0 & -1
\end{array}\right)_{A B}
\end{aligned}
$$

To perform the calculation one employs the propagator given in Eq. (43) with $\Delta_{\text {eff }}$ given in (51) and the interaction vertices in (73). The result of the calculation of the two diagrams at the second order in the momentum expansion is as follows:

$$
\mathcal{L}_{\text {eff }}(p)_{\text {s.e. }}=i \frac{4 \times 4 \mu^{2}}{16 \pi^{3} f^{2}} \sum_{\vec{v}} \sum_{m, k=1}^{8} \frac{1}{2}\left(\frac{\pi \Delta}{R}\right)^{2} \delta_{R}\left[h\left(\vec{v} \cdot \vec{n}_{m}\right)\right]\left(i \varphi^{(m)}\right)
$$




$$
\begin{aligned}
\times & \delta_{R}\left[h\left(\vec{v} \cdot \vec{n}_{k}\right)\right]\left(i \varphi^{(k)}\right) \int \frac{d^{2} \ell}{D(\ell) D(\ell+p)} \\
& \times\left[-2 \Delta_{e f f}^{2}+V \cdot \ell \tilde{V} \cdot(\ell+p)+\tilde{V} \cdot \ell V \cdot(\ell+p)\right], \\
\mathcal{L}_{e f f}(p)_{t a d} & =i \frac{4 \times 4 \mu^{2}}{16 \pi^{3} f^{2}} \sum_{\vec{v}} \sum_{m=1}^{8} \int \frac{d^{2} \ell}{D(\ell)} \frac{\pi \Delta \Delta_{e f f}}{R} \delta_{R}\left[h\left(\vec{v} \cdot \vec{n}_{m}\right)\right]\left(\varphi^{(m)}\right)^{2}
\end{aligned}
$$

where

$$
D(\ell)=\ell_{0}^{2}-\ell_{\|}^{2}-\Delta_{e f f}^{2}+i \epsilon,
$$

One can easily control that the Goldstone theorem is satisfied and the phonons are massless. As a matter of fact one has

$$
\begin{aligned}
\mathcal{L}_{\text {mass }} & =\mathcal{L}_{\text {eff }}(0)_{\text {s.e. }}+\mathcal{L}_{\text {eff }}(0)_{\text {tad }}=i \frac{4 \times 4 \mu^{2}}{16 \pi^{3} f^{2}} \frac{\pi \Delta}{R} \times \\
& \times \sum_{\vec{v}} \int \frac{d^{2} \ell}{D(\ell)}\left[-\sum_{m, k=1}^{8} \frac{\pi \Delta}{R} \delta_{R}\left[h\left(\vec{v} \cdot \vec{n}_{m}\right)\right] \varphi^{(m)} \delta_{R}\left[h\left(\vec{v} \cdot \vec{n}_{k}\right)\right] \varphi^{(k)}\right. \\
& \left.+\Delta_{\text {eff }} \sum_{m=1}^{8} \delta_{R}\left[h\left(\vec{v} \cdot \vec{n}_{m}\right)\right]\left(\varphi^{(m)}\right)^{2}\right]
\end{aligned}
$$

Both terms in the r.h.s. of eq. (76) present a double sum: $m, k=1, \ldots, 8$ (see eq. (51) for $\Delta_{\text {eff }}$ ); we have seen at the end of section 2 that the eight rings that define the pairing region are non overlapping. Therefore only the terms with $m=k$ survive and one immediately verifies the validity of the Goldstone's theorem. i.e. the vanishing of (76). Notice that in our approximation the masses of the Goldstone bosons vanish only if the pairing regions are not overlapping, signaling that when the regions overlap we are not at the minimum of the free-energy (see [11]).

At the second order in the momentum expansion one has

$$
\begin{aligned}
\mathcal{L}_{e f f}(p) & =i \frac{4 \times 4 \mu^{2}}{16 \pi^{3} f^{2}} \sum_{\vec{v}} \sum_{m, k=1}^{8} \frac{1}{2}\left(\frac{\pi \Delta}{R}\right)^{2} \delta_{R}\left[h\left(\vec{v} \cdot \vec{n}_{m}\right)\right]\left(i \varphi^{(m)}\right) \\
& \times \delta_{R}\left[h\left(\vec{v} \cdot \vec{n}_{k}\right)\right]\left(i \varphi^{(k)}\right) \int d^{2} \ell \frac{2 \Delta_{e f f}^{2} V \cdot p \tilde{V} \cdot p}{[D(\ell)]^{3}} .
\end{aligned}
$$

Using the result

$$
\int \frac{d^{2} \ell}{[D(\ell)]^{3}}=-\frac{i \pi}{2 \Delta_{e f f}^{4}}
$$


and the absence of off-diagonal terms in the double sum, we get the effective lagrangian in the form

$$
\begin{aligned}
\mathcal{L}_{e f f}(p) & =-\frac{\mu^{2}}{2 \pi^{2} f^{2}} \sum_{\vec{v}}\left(\frac{\pi \Delta}{R}\right)^{2} \sum_{k=1}^{8} \frac{\left(\delta_{R}\left[h\left(\vec{v} \cdot \vec{n}_{k}\right)\right]\right)^{2}}{\Delta_{e f f}^{2}} \\
& \times(V \cdot p) \varphi^{(k)}(\tilde{V} \cdot p) \varphi^{(k)} .
\end{aligned}
$$

To perform the calculation one can exploit the large value found per $R$, i.e. eq. (54). Since for $R \rightarrow \infty$ the $\delta_{R}$ function becomes the Dirac delta, we take the $R \rightarrow \infty$ limit and we handle the $\delta_{R}$ function according to the Fermi trick in the Golden Rule; in the numerator we substitute one $\delta_{R}$ function with the Dirac delta while for the other one we take

$$
\frac{\pi \delta_{R}[h(x)]}{R} \rightarrow \frac{\pi \delta_{R}(0)}{R} \rightarrow 1 .
$$

We observe that this limit is justified by the large value found for $R$, see eq. (54). The sum over $k$ in $(79)$ gives

$$
\begin{aligned}
& \sum_{k=1}^{8} \delta_{R}\left[h\left(\vec{v} \cdot \vec{n}_{k}\right)\right] \varphi^{(k)} \delta_{R}\left[h\left(\vec{v} \cdot \vec{n}_{k}\right)\right] \varphi^{(k)} \rightarrow \frac{R}{\pi} \sum_{k=1}^{8} \delta\left[h\left(\vec{v} \cdot \vec{n}_{k}\right)\right]\left(\varphi^{(k)}\right)^{2}= \\
& =\frac{R}{\pi} \sum_{k=1}^{8} \delta\left[1-\frac{\delta \mu}{q \vec{v} \cdot \vec{n}_{k}}\right]\left(\varphi^{(k)}\right)^{2}=\frac{R^{2}}{\pi^{2}} k_{R} \sum_{k=1}^{8} \delta\left[\vec{v} \cdot \vec{n}_{k}-\frac{\delta \mu}{q}\right]\left(\varphi^{(k)}\right)^{2}(81)
\end{aligned}
$$

with

$$
k_{R}=\frac{\pi|\delta \mu|}{q R} .
$$

On the other hand $\Delta_{\text {eff }}^{2}$ in the denominator of (79) gives

$$
\Delta_{\text {eff }}^{2} \rightarrow \Delta^{2}
$$

Therefore one gets

$$
\mathcal{L}_{e f f}(p)=-\frac{\mu^{2} k_{R}}{2 \pi^{2} f^{2}} \sum_{i, j=1}^{3} \sum_{k=1}^{8} \sum_{\vec{v}} \delta\left\{\vec{v} \cdot \vec{n}_{k}-\frac{\delta \mu}{q}\right\} V_{\mu} \tilde{V}_{\nu} \epsilon_{i}^{(k)} \epsilon_{j}^{(k)} p_{\mu} \phi^{(i)} p_{\nu} \phi^{(j)} .
$$

The integration over the Fermi velocities require special attention. We use the result

$$
\sum_{k=1}^{8} \epsilon_{i}^{(k)} \epsilon_{j}^{(k)}=8 \delta_{i j}
$$


this fixes the constant multiplying the time derivative term in the effective lagrangian at the value (taking into account (10))

$$
\frac{8 \mu^{2} k_{R}}{4 \pi^{2} f^{2}}
$$

Therefore one obtains canonical normalization for the kinetic term provided

$$
f^{2}=\frac{8 \mu^{2} k_{R}}{2 \pi^{2}}
$$

To compute the parameters $a, b, c$ of the effective lagrangian (69) we need to evaluate

$$
\beta_{\ell m}^{i j}=\sum_{k=1}^{8} \epsilon_{i}^{(k)} \epsilon_{j}^{(k)} \int \frac{d \vec{v}}{8 \pi} v_{\ell} v_{m} \delta\left\{\vec{v} \cdot \vec{n}_{k}-\frac{\delta \mu}{q}\right\} .
$$

We find the result (see Appendix 2)

$$
\beta_{\ell m}^{i j}=\frac{2}{3} \delta_{l m} \delta_{i j}+\frac{3 \cos ^{2} \theta_{q}-1}{3} \sum_{r=1}^{3} \rho_{l m}^{r} \rho_{i j}^{r}
$$

where

$$
\rho_{i j}^{r}=\delta_{i s} \delta_{j t}+\delta_{i t} \delta_{j s}, \quad r, s, t \text { in cyclic order . }
$$

From this equation we get

$$
\begin{aligned}
\mathcal{L}_{\text {eff }}(p)=\frac{1}{2}\left(p^{0^{2}} \phi^{(i)^{2}}-\frac{1}{8} \beta_{l m}^{i j} p^{l} p^{m} \phi^{(i)} \phi^{(j)}\right)= \\
=\frac{1}{2}\left(p^{0^{2}} \phi^{(i)^{2}}-\frac{|\vec{p}|^{2}}{12} \phi^{(i)^{2}}-\frac{3 \cos ^{2} \theta_{q}-1}{6} \sum_{i<j=1,2,3} p^{i} \phi^{(i)} p^{j} \phi^{(j)}\right) .
\end{aligned}
$$

Comparing with eq. (69) we finally obtain

$$
a=\frac{1}{12}, \quad b=0, \quad c=\frac{3 \cos ^{2} \theta_{q}-1}{12} .
$$

One can note that these values for the parameters of the phonon effective lagrangian satisfy the positivity constraints found in [23].

In [23] one can find the general discussion of the dispersion relation for arbitrary values of the parameters $a, b$ and $c$. In the present paper we give the expression for the eigenvalues of $E^{2}$, according to the direction of propagation of the momentum $\vec{p}$, in table 1 . 


\begin{tabular}{|c|c|c|}
\hline Momentum & Eigenvalues & Eigenvectors \\
\hline$\vec{p}=p \vec{e}^{(i)}$ & $\begin{array}{l}1 / 12 \\
1 / 12\end{array}$ & $\begin{array}{c}\phi^{(i)} \\
\phi^{(j)}, \phi^{(k)}(i \neq j \neq k)\end{array}$ \\
\hline$\vec{p}=p\left(\vec{e}^{(1)}+\vec{e}^{(2)}+\vec{e}^{(3)}\right)$ & $\begin{array}{l}\left(1+3 \sin ^{2} \theta_{q}\right) / 6 \\
\left(1+6 \cos ^{2} \theta_{q}\right) / 36\end{array}$ & $\begin{array}{c}-\phi^{(1)}+x \phi^{(2)}+(1-x) \phi^{(3)} \\
\phi^{(1)}+\phi^{(2)}+\phi^{(3)}\end{array}$ \\
\hline$\vec{p}=p\left(-\vec{e}^{(1)}+\vec{e}^{(2)}+\vec{e}^{(3)}\right)$ & $\begin{array}{c}\left(1+3 \sin ^{2} \theta_{q}\right) / 6 \\
\left(1+6 \cos ^{2} \theta_{q}\right) / 36\end{array}$ & $\begin{array}{c}\phi^{(1)}+x \phi^{(2)}+(1-x) \phi^{(3)} \\
-\phi^{(1)}+\phi^{(2)}+\phi^{(3)}\end{array}$ \\
\hline$\vec{p}=p\left(\vec{e}^{(1)}-\vec{e}^{(2)}+\vec{e}^{(3)}\right)$ & $\begin{array}{l}\left(1+3 \sin ^{2} \theta_{q}\right) / 6 \\
\left(1+6 \cos ^{2} \theta_{q}\right) / 36\end{array}$ & $\begin{array}{c}\phi^{(1)}+x \phi^{(2)}-(1-x) \phi^{(3)} \\
\phi^{(1)}-\phi^{(2)}+\phi^{(3)}\end{array}$ \\
\hline$\vec{p}=p\left(\vec{e}^{(1)}+\vec{e}^{(2)}-\vec{e}^{(3)}\right)$ & $\begin{array}{l}\left(1+3 \sin ^{2} \theta_{q}\right) / 6 \\
\left(1+6 \cos ^{2} \theta_{q}\right) / 36\end{array}$ & $\begin{array}{c}\phi^{(1)}+x \phi^{(2)}+(1+x) \phi^{(3)} \\
\phi^{(1)}+\phi^{(2)}-\phi^{(3)}\end{array}$ \\
\hline $\begin{array}{c}\vec{p}=p\left(\vec{e}^{(i)}+\vec{e}^{(j)}\right) \\
i<j\end{array}$ & $\begin{array}{c}1 / 12 \\
\sin ^{2} \theta_{q} / 8 \\
\left(1+3 \cos ^{2} \theta_{q}\right) / 24\end{array}$ & $\begin{array}{c}\phi^{(k)} \\
-\phi^{(i)}+\phi^{(j)} \\
\phi^{(i)}+\phi^{(j)}\end{array}$ \\
\hline $\begin{array}{c}\vec{p}=p\left(\vec{e}^{(i)}-\vec{e}^{(j)}\right) \\
i<j\end{array}$ & $\begin{array}{c}1 / 12 \\
\sin ^{2} \theta_{q} / 8 \\
\left(1+3 \cos ^{2} \theta_{q}\right) / 24\end{array}$ & $\begin{array}{c}\phi^{(k)} \\
\phi^{(i)}+\phi^{(j)} \\
-\phi^{(i)}+\phi^{(j)}\end{array}$ \\
\hline
\end{tabular}

Table 1 - Eigenvalues and eigenvectors of $\frac{E^{2}}{|\vec{p}|^{2}} ; \vec{p}$ along the symmetry axes. 


\section{Gluon dynamics in the the LOFF phase: single plane wave structure}

In this Section and in the subsequent one we wish to derive the effective lagragian for the gluons of the unbroken $S U(2)_{c}$ subgroup of the two-flavor LOFF phase. To start with we review the results obtained for the gluon effective lagrangian at one-loop in the $2 S C$ isotropic phase [5]. They were obtained in 25]; in 26] the results of [25] were obtained by the method of the High Density Effective Theory and extended to the three-gluon and four-gluon vertices. In the $2 S C$ phase one considers massless quarks of two different flavors at the same chemical potential $\mu$ and at low temperature. Color superconductivity breaks the color group $S U(3)_{c}$ down to a $S U(2)_{c}$ subgroup and the quarks with nontrivial $S U(2)_{c}$ charges acquire a gap $\Delta_{0}$. Therefore only the three gluons relative to the generators of the unbroken subgroup remain massless.

The lagrangian including one loop corrections for the $S U(2)_{c}$ gluons can be written as 25], 26]

$$
\mathcal{L}=\frac{1}{2}\left(E_{i}^{a} E_{i}^{a}-B_{i}^{a} B_{i}^{a}\right)+\frac{k}{2} E_{i}^{a} E_{i}^{a},
$$

with $a=1,2,3$ color indices and

$$
k=\frac{g^{2} \mu^{2}}{18 \pi^{2} \Delta_{0}^{2}} .
$$

Eqns. (93) and (94) mean that, at one loop, the medium has a dielectric constant

$$
\epsilon=k+1
$$

and a magnetic permeability $\lambda=1$, while the gluon speed in the medium is

$$
v=\frac{1}{\sqrt{\epsilon \lambda}} \propto \frac{\Delta_{0}}{g \mu} .
$$

As noted in [25], it is possible to put (93) in a gauge invariant form

$$
\mathcal{L}=-\frac{1}{4} F_{a}^{\mu \nu} F_{\mu \nu}^{a} \quad(a=1,2,3),
$$

provided the following rescaling is used

$$
A_{0}^{a} \rightarrow A_{0}^{a \prime}=k^{3 / 4} A_{0}^{a},
$$




$$
\begin{gathered}
A_{i}^{a} \rightarrow A_{i}^{a \prime}=k^{1 / 4} A_{i}^{a}, \\
x_{0} \rightarrow x_{0}^{\prime}=k^{-1 / 2} x_{0}, \\
g \rightarrow g^{\prime}=k^{-1 / 4} g .
\end{gathered}
$$

Let us now consider the LOFF non-isotropic phase. To begin with we assume the crystal structure given by a plane wave i.e. $\Delta(\vec{x})$ given by (42). The difference with the isotropic case consists, in the present case, in the substitution

$$
\Delta \rightarrow \Delta_{e f f}=\frac{\Delta \pi}{R} \delta_{R}[h(\vec{v} \cdot \vec{n})],
$$

where $\vec{n}=\vec{q} / q$ and $h(x)$ given by (30).

The effective action allows the evaluation of the one loop diagrams with two external gluon lines and internal quark lines similar to those in fig. 2. Their contribution to the polarization tensor is written down in Appendix 3.

Let us now write

$$
\Pi_{a b}^{\mu \nu}(p)=\Pi_{a b}^{\mu \nu}(0)+\delta \Pi_{a b}^{\mu \nu}(p) .
$$

As discussed in Appendix 3 we find a vanishing Meissner mass

$$
\Pi_{a b}^{i j}(0)=0
$$

and a non-vanishing Debye screening mass

$$
m_{D}=\frac{g \mu}{\pi} \sqrt{1+\frac{\cos \theta_{a}-\cos \theta_{b}}{2}},
$$

where $\cos \theta_{a}$ and $\cos \theta_{b}\left(-1 \leq \cos \theta_{a} \leq \cos \theta_{b} \leq 1\right)$ are the solutions of the equation

$$
|h(\cos \theta)|=\frac{\pi}{2 R} .
$$

where $h(\cos \theta)$ is defined in eq. (291).

It is interesting to note that, in absence of LOFF pairing, i.e. if the pairing region vanishes and $\cos \theta_{a}=\cos \theta_{b}$, one gets for the Meissner and Debye mass the same results obtained in the hard dense loop approximation for the model with two flavors in the normal (not superconducting) state, see e.g. [27]. On the other hand if the blocking region vanishes $\left(\cos \theta_{a}=-1\right.$, $\cos \theta_{b}=+1$ ) one is in the 2SC phase and eq. (105) gives $m_{D}=0$ as expected.

Next consider $\delta \Pi_{a b}^{\mu \nu}(p)$. The contribution of the blocking region vanishes as discussed in Appendix 3. Therefore the only non vanishing contribution 
to $\delta \Pi_{a b}^{\mu \nu}(p)$ comes from the pairing region. It could be computed exactly, but the corresponding expressions are cumbersome and shed little light on the underlying physics. Therefore we prefer to work in the approximation of small momenta $(|p| \ll \Delta)$. We find

$$
-\delta \Pi_{a b}^{\mu \nu}(p)=\delta_{a b} \frac{\mu^{2} g^{2}}{12 \pi^{2}} \sum_{\vec{v} ; \text { pairing }} \frac{V^{\mu} V^{\nu}(\tilde{V} \cdot p)^{2}-\tilde{V}^{\mu} V^{\nu}(V \cdot p \tilde{V} \cdot p)+V \leftrightarrow \tilde{V}}{\Delta_{\text {eff }}^{2}} .
$$

That is

$$
\begin{aligned}
-\delta \Pi_{a b}^{00}(p) & =\delta_{a b} \frac{g^{2} \mu^{2}}{3 \pi^{2}} \sum_{\vec{v} ; \text { pairing }} \frac{v_{i} v_{j}}{\Delta_{\text {eff }}^{2}} p_{i} p_{j}= \\
& =\delta_{a b} \frac{g^{2} \mu^{2} R^{2}}{3 \Delta^{2} \pi^{4}} \int_{\text {pairing }} \frac{d \cos \theta d \phi}{8 \pi} \frac{v_{i} v_{j}}{\left(\delta_{R}[h(\cos \theta)]\right)^{2}} p_{i} p_{j}
\end{aligned}
$$

where $\vec{v}=(\sin \theta \cos \phi, \sin \theta \sin \phi, \cos \theta)$ and $\vec{n}=\vec{q} / q$ along the $z$-axis. The integration domain is defined by $\cos \theta_{a}<\cos \theta<\cos \theta_{b}$. We get therefore

$$
-\delta \Pi_{a b}^{00}(p)=\delta_{a b} k\left(f(R) p_{\perp}^{2}+g(R) p_{\|}^{2}\right),
$$

where $k$ is given by (94) and

$$
\begin{gathered}
f(R)=\frac{3}{4} \int_{\text {pairing }} d \cos \theta\left(1-\cos ^{2} \theta\right), \\
g(R)=\frac{3}{2} \int_{\text {pairing }} d \cos \theta \cos ^{2} \theta
\end{gathered}
$$

are functions of the parameter $R$ and are reported in fig. $3(q \approx 1.2|\delta \mu|)$.

It is interesting to note the anisotropy of the polarization tensor exhibited by these results. One has always $g>f$; for large $R$, and neglecting $\delta \mu / \mu$ corrections, one finds approximately

$$
\frac{g(R)}{f(R)} \rightarrow \frac{2}{\left(\frac{q}{\delta \mu}\right)^{2}-1}
$$

Let us finally write down the remaining components of the polarization tensor. From (107) we get

$-\Pi_{a b}^{i j}(p)=\delta_{a b} \frac{g^{2} \mu^{2}}{3 \pi^{2}} \sum_{\vec{v} ; \text { pairing }} \frac{v_{i} v_{j}}{\Delta^{2}} p_{0}^{2}=k p_{0}^{2}\left(f(R)\left(\delta_{i 1} \delta_{j 1}+\delta_{i 2} \delta_{j 2}\right)+g(R) \delta_{i 3} \delta_{j 3}\right)$ 

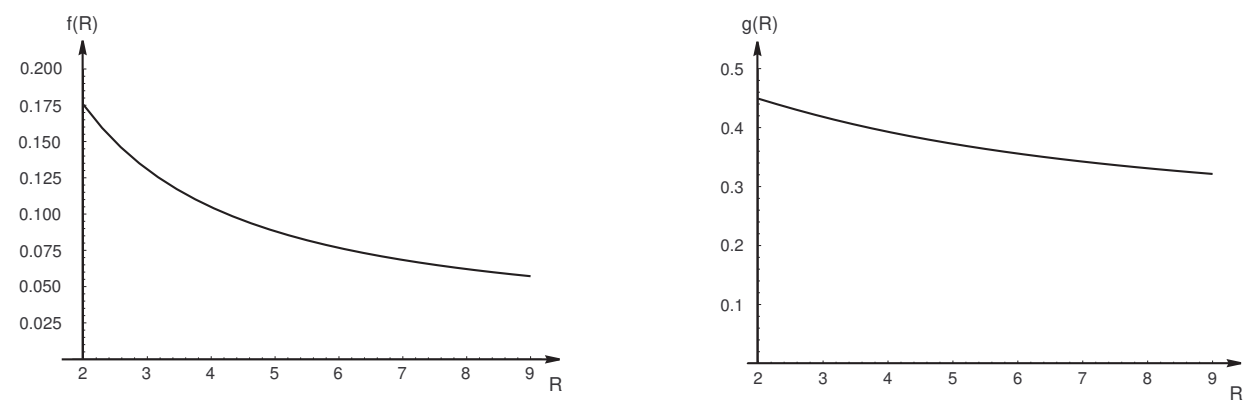

Fig. 3 Plots of the functions $f(R)$ and $g(R)$.

and

$$
-\Pi_{a b}^{0 i}(p)=k p_{0} p^{j}\left(f(R)\left(\delta_{i 1} \delta_{j 1}+\delta_{i 2} \delta_{j 2}\right)+g(R) \delta_{i 3} \delta_{j 3}\right)
$$

These results complete the analysis of the LOFF model in the one plane wave approximation. From $\Pi_{a b}^{\mu \nu}$ we get the dispersion law for the gluons at small momenta. The lagrangian at one loop is

$$
\mathcal{L}=-\frac{1}{4} F_{a}^{\mu \nu} F_{\mu \nu}^{a}-\frac{1}{2} \Pi_{a b}^{\mu \nu} A_{\mu}^{a} A_{\nu}^{b}
$$

(sum over the repeated color indices $a, b=1,2,3)$. Introducing the fields $E_{i}^{a} \equiv F_{0 i}^{a}$ and $B_{i}^{a} \equiv i \varepsilon_{i j k} F_{j k}^{a}$, and using (109), (113) and (114) we can rewrite the lagrangian (115) as follows

$$
\mathcal{L}=\frac{1}{2}\left(\epsilon_{i j} E_{i}^{a} E_{j}^{a}-B_{i}^{a} B_{i}^{a}\right)+\frac{1}{2} m_{D}^{2} A_{a}^{0} A_{a}^{0},
$$

where

$$
\epsilon_{i j}=\left(\begin{array}{ccc}
1+k f(R) & 0 & 0 \\
0 & 1+k f(R) & 0 \\
0 & 0 & 1+k g(R)
\end{array}\right) .
$$

This means that the medium has a non-isotropic dielectric tensor $\epsilon$ and a magnetic permeability $\lambda=1$. These results have been obtained taking the

${ }^{2}$ We do not include here the 3 and 4 -gluon vertices that however can be handled as in [26], with the result that the local gauge invariance of the one-loop lagrangian is satisfied. 
total momentum of the Cooper pairs along the $z$ direction. Therefore we distinguish the dielectric constant along the $z$ axis, which is

$$
\epsilon_{\|}=1+k g(R)
$$

and the dielectric constant in the plane perpendicular to the $z$ axis

$$
\epsilon_{\perp}=1+k f(R)
$$

This means that the gluon speed in the medium depends on the direction of propagation of the gluon; along the $z$ axis the gluon velocity is

$$
v_{\|} \simeq \frac{1}{\sqrt{k g(R)}},
$$

while for gluons which propagate in the $x-y$ plane we have

$$
v_{\perp} \simeq \frac{1}{\sqrt{k f(R)}}
$$

and in the limit of large $R$, and neglecting $\delta \mu / \mu$ corrections,

$$
v_{\|} \rightarrow \frac{1}{\sqrt{2}} \tan \theta_{q} v_{\perp}
$$

with $\cos \theta_{q}$ defined in eq. (30). The anisotropy in the gluon velocities has its pendant in the anisotropy of the phonon velocity [21].

\section{Gluon dynamics in the LOFF phase: cubic structure}

The condensate in this case is given by eq. (45), so that we will use the results of sec. 2 with $\Delta_{\text {eff }}$ given by (51). The calculations are similar to the previous case and, similarly, the $S U(2)_{c}$ gluons have vanishing Meissner mass and exhibit partial Debye screening. However the dispersion law of the gluons is different.

As a matter of fact we write the one loop lagrangian for the $S U(2)_{c}$ gluons as

$$
\mathcal{L}=\frac{1}{2}\left(E_{i}^{a} E_{i}^{a}-B_{i}^{a} B_{i}^{a}\right)+\delta \mathcal{L},
$$


with

$$
\delta \mathcal{L}=-\frac{1}{2} \Pi_{a b}^{\mu \nu} A_{\mu}^{a} A_{\nu}^{b} .
$$

In the approximation $|p| \ll \Delta, \delta \Pi_{a b}^{\mu \nu}$ is again given by eq. (107), but now $\Delta_{e f f}$ is given by (51). One gets

$$
\delta \mathcal{L} \equiv E_{i}^{a} E_{j}^{b} \delta_{a b} \frac{g^{2} \mu^{2}}{6 \pi^{2}} \int_{\text {pairing }} \frac{d \cos \theta d \phi}{8 \pi} \frac{v_{i} v_{j}}{\Delta_{\text {eff }}^{2}}+A_{0}^{a} A_{0}^{b} \delta_{a b} \frac{g^{2} \mu^{2}}{4 \pi^{2}} \int_{\text {blocking }} d \cos \theta
$$

Evaluating the integrals one finds

$$
\mathcal{L}=\frac{1}{2}\left(\tilde{\epsilon}_{i j} E_{i}^{a} E_{j}^{a}-B_{i}^{a} B_{i}^{a}\right)+\frac{1}{2} M_{D}^{2} A_{0}^{a} A_{0}^{a},
$$

with the tensor $\tilde{\epsilon}^{i j}$ given by

$$
\tilde{\epsilon}_{i j}=\delta_{i j}[1+k t(R)]
$$

and

$$
M_{D}=\frac{g \mu}{\pi} \sqrt{1+8 \frac{\cos \theta_{a}-\cos \theta_{b}}{2}} .
$$

where $\cos \theta_{a, b}$ are solutions of eq. (106). It should also be noted that the values of the parameter $R$ for the cube and the plane wave can be different.

The isotropy of these results can be easily understood noticing that $\delta \mathcal{L}$ is a symmetric function of its arguments. As a matter of fact it must be symmetric under the residual symmetry $O_{h}$ and therefore it must be built up from the three elementary symmetric functions $I_{2}\left(X_{i}\right), I_{4}\left(X_{i}\right)$ and $I_{6}\left(X_{i}\right)$, defined in eq. (66), with $X_{i}=E_{i}^{a}$. The invariance of $\delta \mathcal{L}$ follows from the invariance of these functions under the three elementary rotations $R_{i}$ and the inversion $I$. However it is clear from (125) that $\delta \mathcal{L}$ is second-order in $E_{a}^{i}$ and therefore the only function that can be involved is $I_{2}$. In conclusion in the cubic lattice one necessarily gets

$$
\tilde{\epsilon}_{i j}-\delta_{i j}=A \delta_{i j}
$$

From eqs. (125) and (51) one has that the function $t(R)$ is given by the formula

$$
t(R) \delta_{i j}=\frac{3 R^{2}}{4 \pi^{3}} \int_{\text {pairing }} d \cos \theta d \phi \frac{v_{i} v_{j}}{\left[\sum_{k=1}^{8} \delta_{R}\left(h\left(\vec{v} \cdot \vec{n}_{k}\right)\right)\right]^{2}}
$$


$t(R)$ can be computed by observing that, using the properties of the $\delta_{R}$ function

$$
\begin{aligned}
t(R) \delta_{i j} & \approx \frac{3 R^{2}}{4 \pi^{3}} \sum_{k=1}^{8} \int_{\text {pairing }} d \cos \theta d \phi \frac{v_{i} v_{j}}{\left[\delta_{R}\left(h\left(\vec{v} \cdot \vec{n}_{k}\right)\right)\right]^{2}}= \\
& =\sum_{k=1}^{8}\left(f(R) \delta_{i j}+[g(R)-f(R)] n_{i}^{(k)} n_{j}^{(k)}\right) .
\end{aligned}
$$

Using (85) we finally get

$$
t(R)=\frac{8}{3}[2 f(R)+g(R)] .
$$

A plot of the function $t(R)$ is in Fig. 4 .

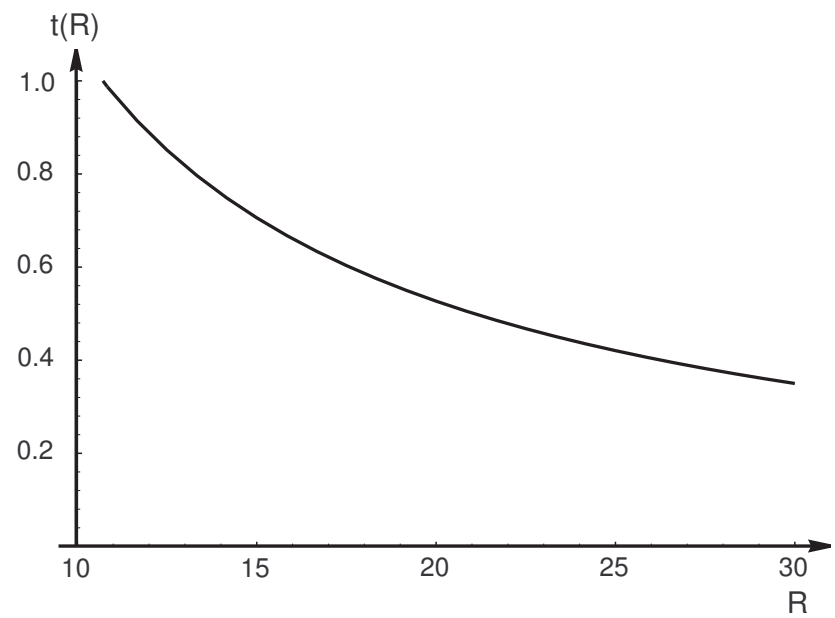

Fig. 4 Plot of the function $t(R)$.

We finally note that, even if the crystalline structure is not isotropic, in eq. (126) the $O(3)$ invariance is restored. This happens because the tensor $\tilde{\epsilon}_{i j}$ is proportional to the identity. Therefore the dielectric properties of the medium will be isotropic and the velocity of propagation of the gluons will be the same in all the directions. We stress that these results are valid in the approximation of soft gluon momenta. 


\section{Conclusions}

We have used the High Density Effective Theory (HDET) formalism to calculate the low energy properties of phonons and gluons in the LOFF phase of QCD.The HDET formalism is very useful to simplify the dynamics as it allows direct elimination of the degrees of freedom which are far from the Fermi surface. It bears useful resemblances with the Heavy Quark Effective Theory of general use when dealing with heavy hadrons. We have first made the modification for the case of a single plane wave condensate and we have derived the quark propagators. After that we have gone to the relevant case of the cubic strucure of ref. [1] corresponding to the sum of eight plane waves. We have added the suitable term in the Lagrangian bearing the sum over the eight plane waves of the cubic structure and derived for this more complex case the quark propagators to be used in the calculations.

The three phonons correspond to the local deformations from the fluctuations of the crystal as defined by the cubic condensate. They are the Nambu-Goldstone bosons from the breaking of translational symmetry. We have studied the low energy limit, where wavelengths are much longer than lattice spacing, and built up an effective lagrangian for the phonons, which, at the second order in the derivatives, depends on three parameters, in agreement with the general theory of the elastic waves in crystals 24.

The parameters of the effective phonon lagrangian are calculated by introducing external fields with the phonon quantum numbers and performing the derivative expansion of the generating functional. The phonons remain massless, as expected. The final results for the parameters are remarkably simple, the only quantity appearing in the result being the ratio of $\delta \mu$ to the common length of the eight wave vectors appearing in the condensate.

We have also derived the effective lagrangian of the gluons of the unbroken $S U(2)_{c}$ subgroup. The gluon self energy diagrams at one loop can be evaluated from the derived effective action. The Meissner mass vanishes as in the case of complete isotropy; on the other hand there is a partial Debye screening originating from the existence of blocking regions on the Fermi spheres. For soft gluon momenta, in the case of a single plane wave, the dielectric constant of the medium is non isotropic and it takes on different values according to its direction; also the gluon velocity is anisotropic. In the case of the cubic crystal the gluon dispersion law for small momenta is however isotropic. It is interesting to remark that at least for gluon long wavelengths, the essential anisotropy of the cubic structure is washed out, 
while it remains visible for the plane wave crystal structure.

\section{Acknowledgements}

Two of us, R.C. and G. N., wish to thank the DESY theory group, where this work was completed, for the very kind hospitality.

\section{Appendix 1: The cube symmetry group $O_{h}$}

The cube symmetry group $O_{h}$ is the finite group containing the transformations that leave the cube (see Fig. 1) invariant. It is defined as follows:

$$
O_{h}=O \times C_{i}
$$

where $O$ is the octahedron group (see below) and $C_{i}=\{E, I\}$ is the center of symmetry of the group, containing the neutral element $E$ and the inversion $I$ :

$$
E=\left(\begin{array}{lll}
1 & 0 & 0 \\
0 & 1 & 0 \\
0 & 0 & 1
\end{array}\right), \quad I=\left(\begin{array}{ccc}
-1 & 0 & 0 \\
0 & -1 & 0 \\
0 & 0 & -1
\end{array}\right)
$$

i.e.

$$
\begin{aligned}
E & : \quad x_{1} \rightarrow x_{1}, \quad x_{2} \rightarrow x_{2}, \quad x_{3} \rightarrow x_{3} \\
I: & x_{1} \rightarrow-x_{1}, \quad x_{2} \rightarrow-x_{2}, \quad x_{3} \rightarrow-x_{3} .
\end{aligned}
$$

The group $O_{h}$ contains 48 elements, comprising the 24 elements of the octahedron group $O$ and other 24 given by $g \cdot I(g \in O)$. The octahedron group is defined by the following system of axes of symmetry:

- Three 4-fold axes 3 , denoted as $C_{4}$ in Fig. 1;

- four 3-fold axes, denoted as $C_{3}$ in Fig. 1;

- six 2-fold axes, denoted as $C_{2}$ in Fig. 1.

Its 24 elements are divided into five classes:

\footnotetext{
${ }^{3}$ For any crystal a $n$-fold axis is such that the crystal assumes the same position after a rotation of an angle $2 \pi / n$ around it. Such a rotation is denoted as $C_{n}$; for extension we denote by $C_{n}$ not only the rotations but also the corresponding symmetry axes.
} 
- Class I, containing only $E$;

- class II, containing three rotations $C_{4}$ and three rotations $C_{4}^{3}$ around the three 4 -fold axes ${ }^{4}$;

- class III containing three rotations $C_{4}^{2}$ three rotations of $2 \times 2 \pi / 4=\pi$ around the three 4-fold axes;

- class IV, containing four rotations $C_{3}$ and four rotations $C_{3}^{2}$ around the four 3-fold axes;

- class $\mathrm{V}$, containing six rotations $C_{2}$ around the six 2-fold axes;

It is easy to construct all the 24 group elements. Class II comprises the three $C_{4}$ rotations, i.e. rotations of $\pi / 2$ around the three 4 -fold axis:

$$
\mathbf{C}_{4}: \quad R_{1}=\left(\begin{array}{ccc}
1 & 0 & 0 \\
0 & 0 & 1 \\
0 & -1 & 0
\end{array}\right), \quad R_{2}=\left(\begin{array}{ccc}
0 & 0 & -1 \\
0 & 1 & 0 \\
1 & 0 & 0
\end{array}\right), \quad R_{3}=\left(\begin{array}{ccc}
0 & 1 & 0 \\
-1 & 0 & 0 \\
0 & 0 & 1
\end{array}\right)
$$

and the three $C_{4}^{3}$ rotations:

$$
\mathrm{C}_{4}^{3}: \quad R_{1}^{3}, \quad R_{2}^{3}, \quad R_{3}^{3}
$$

Class III comprises the three $C_{4}^{2}$ rotations:

$$
\mathrm{C}_{4}^{2}: \quad R_{1}^{2}, \quad R_{2}^{2}, \quad R_{3}^{2}
$$

Class IV comprises four $C_{3}$ rotations:

$$
\mathrm{C}_{3}: \quad A_{1}=R_{1} R_{2}, \quad A_{2}=R_{1} A_{1} R_{1}^{-1}, \quad A_{3}=R_{2} A_{1} R_{2}^{-1}, \quad A_{4}=A_{3} A_{1} A_{3}^{-1}
$$

and four $C_{3}^{2}$ rotations:

$$
\mathbf{C}_{3}^{2}: \quad A_{1}^{2}, \quad A_{2}^{2}, \quad A_{3}^{2}, \quad A_{4}^{2} ;
$$

finally class $\mathrm{V}$ comprises six $C_{2}$ rotations:

$$
\mathrm{C}_{2}: \quad B_{1}=A_{1} R_{2}, \quad B_{2}=A_{1} B_{1} A_{1}^{-1}, \quad B_{3}=A_{2} B_{1} A_{2}^{-1},
$$

\footnotetext{
${ }^{4}$ In general $C_{n}^{m}$ denotes a rotation of an angle $m \times 2 \pi / n$ around a n-fold axis; clearly $C_{n}^{n}=1$.
} 


$$
B_{4}=A_{3} B_{1} A_{3}^{-1}, \quad B_{5}=A_{4} B_{1} A_{4}^{-1}, \quad B_{6}=R_{1} B_{1} R_{1}^{-1} .
$$

We finally observe that from the general theorem for finite groups asserting that the sum of the squares of the dimension of the representations, $n_{i}$, must be equal to the order of the group (in this case 24):

$$
\sum_{i} n_{i}^{2}=24
$$

the only possible solution for $O$ is

$$
n_{1}=n_{2}=1, \quad n_{3}=2, \quad n_{4}=n_{5}=3 .
$$

\section{Appendix 2}

In this Appendix we evaluate the coefficients appearing in eq. (88):

$$
\beta_{\ell m}^{i j}=\sum_{k=1}^{8} \epsilon_{i}^{(k)} \epsilon_{j}^{(k)} \int \frac{d \vec{v}}{8 \pi} v_{\ell} v_{m} \delta\left\{\vec{v} \cdot \vec{n}_{k}-\frac{\delta \mu}{q}\right\} .
$$

To this end we make use of the result

$$
\begin{gathered}
\sum_{\vec{v}} v_{i} v_{j} \delta\left\{\cos \theta-\frac{\delta \mu}{q}\right\} \equiv \int \frac{d \vec{v}}{8 \pi} v_{i} v_{j} \delta\left\{\cos \theta-\frac{\delta \mu}{q}\right\}= \\
=\left(\frac{\sin ^{2} \theta_{q}}{8}\left(\delta_{i 1} \delta_{j 1}+\delta_{i 2} \delta_{j 2}\right)+\frac{\cos ^{2} \theta_{q}}{4} \delta_{i 3} \delta_{j 3}\right)
\end{gathered}
$$

with

$$
\cos ^{2} \theta_{q}=\left(\frac{\delta \mu}{q}\right)^{2}
$$

We therefore obtain

$$
\beta_{\ell m}^{i j}=\sum_{k=1}^{8} R_{p \ell}^{(k)} R_{q m}^{(k)} C_{i j}^{(k)}\left(\frac{\sin ^{2} \theta_{q}}{8} \delta_{p q}+\frac{3 \cos ^{2} \theta_{q}-1}{8} \delta_{p 3} \delta_{q 3}\right)
$$

where the eight matrices $R^{(k)}$ are rotation matrices that transform the eight vectors $\vec{n}_{k}$ into the unit vector $(0,0,1)$. They are given by

$$
R^{(1)}=\left(\begin{array}{ccc}
\frac{1}{\sqrt{2}} & -\frac{1}{\sqrt{2}} & 0 \\
\frac{1}{\sqrt{6}} & \frac{1}{\sqrt{6}} & -\sqrt{\frac{2}{3}} \\
\frac{1}{\sqrt{3}} & \frac{1}{\sqrt{3}} & \frac{1}{\sqrt{3}}
\end{array}\right)
$$


and, in the notations of the Appendix 1

$$
\begin{aligned}
& R^{(2)}=R^{(1)} R_{3}^{-1}, \quad R^{(3)}=R^{(1)} R_{3}^{2}, \quad R^{(4)}=R^{(1)} R_{3}, \\
& R^{(5)}=R^{(1)} B_{3}, \quad R^{(6)}=R^{(2)} B_{4}, \quad R^{(7)}=R^{(3)} B_{3}, \quad R^{(8)}=R^{(4)} B_{4} \cdot(150)
\end{aligned}
$$

On the other hand the eight matrices $C_{i j}^{(k)}$ are

$$
C_{i j}^{(k)}=\left(\begin{array}{ccc}
1 & \eta_{3}^{(k)} & \eta_{2}^{(k)} \\
\eta_{3}^{(k)} & 1 & \eta_{1}^{(k)} \\
\eta_{2}^{(k)} & \eta_{1}^{(k)} & 1
\end{array}\right)_{i j}
$$

with

$$
\begin{aligned}
& \eta_{1}^{(k)}=(+1,-1,-1,+1,-1,+1,+1,-1) \\
& \eta_{2}^{(k)}=(+1,+1,-1,-1,-1,-1,+1,+1) \\
& \eta_{3}^{(k)}=(+1,-1,+1,-1,+1,-1,+1,-1) .
\end{aligned}
$$

Let us evaluate $\beta_{l m}^{i j}$. We notice that

$$
C_{i j}^{(k)}=\delta_{i j}+\sum_{r=1,2,3} \eta_{r}^{(k)} \rho_{i j}^{r}
$$

where

$$
\rho_{i j}^{r}=\delta_{i s} \delta_{j t}+\delta_{i t} \delta_{j s}, \quad r, s, t \text { in cyclic order }
$$

and

$$
\sum_{k=1, \cdots, 8} \eta_{r}^{(k)}=0, \quad \sum_{k=1, \cdots, 8} \eta_{r}^{(k)} \eta_{s}^{(k)}=8 \delta_{r s} .
$$

Using the orthogonality of the $R^{(k)}$ matrices we can evaluate the first term of the r.h.s. of (148) immediately

$$
\beta_{\ell m}^{i j}=\sin ^{2} \theta_{q} \delta_{l m} \delta_{i j}+\frac{3 \cos ^{2} \theta_{q}-1}{8} \sum_{k=1}^{8} R_{3 \ell}^{(k)} R_{3 m}^{(k)} C_{i j}^{(k)} .
$$

From the very definition of the matrices $R^{(k)}$ we get

$$
R_{i j}^{(k)} n_{j}^{(k)}=\delta_{i 3} \rightarrow R_{3 j}^{(k)}=n_{j}^{(k)}=\frac{\epsilon_{j}^{(k)}}{\sqrt{3}} .
$$


Therefore

$$
\beta_{\ell m}^{i j}=\sin ^{2} \theta_{q} \delta_{l m} \delta_{i j}+\frac{3 \cos ^{2} \theta_{q}-1}{24} \sum_{k=1}^{8} C_{l m}^{(k)} C_{i j}^{(k)} .
$$

Using the expression (153) for $C_{i j}^{(k)}$ we find

$$
\beta_{\ell m}^{i j}=\frac{2}{3} \delta_{l m} \delta_{i j}+\frac{3 \cos ^{2} \theta_{q}-1}{3} \sum_{r=1}^{3} \rho_{l m}^{r} \rho_{i j}^{r} .
$$

\section{Appendix 3}

For the case of a single plane wave we get the following expression for the polarization tensor:

$$
\begin{aligned}
-\Pi_{a b}^{\mu \nu}(p) & =i \delta_{a b} \frac{g^{2} \mu^{2}}{2 \pi^{3}} \sum_{\vec{v}}\left[V^{\mu} V^{\nu} \tilde{I}_{1}(p)+\tilde{V}^{\mu} \tilde{V}^{\nu} I_{1}(p)+\left(V^{\mu} \tilde{V}^{\nu}+V^{\nu} \tilde{V}^{\mu}\right) I_{2}(p)\right] \\
& -i \delta_{a b} \frac{g^{2}}{\pi^{3}} \sum_{\vec{v}} J\left(2 g^{\mu \nu}-\tilde{V}^{\mu} V^{\nu}-\tilde{V}^{\nu} V^{\mu}\right),
\end{aligned}
$$

where

$$
\begin{gathered}
I_{1}(p)=\int d^{2} \ell \frac{V \cdot \ell V \cdot(\ell+p)}{D(\ell+p) D(\ell)}, \\
\tilde{I}_{1}(p)=\int d^{2} \ell \frac{\tilde{V} \cdot \ell \tilde{V} \cdot(\ell+p)}{D(\ell+p) D(\ell)}, \\
I_{2}(p)=\int d^{2} \ell \frac{\Delta_{e f f}^{2}}{D(\ell+p) D(\ell)}, \\
J=\int_{-\mu}^{+\mu} d \ell_{\|}\left(\mu+\ell_{\|}\right)^{2} \int_{-\infty}^{+\infty} d \ell_{0} \frac{\tilde{V} \cdot \ell}{(2 \mu+\tilde{V} \cdot \ell+i \epsilon) D(\ell)},
\end{gathered}
$$

and $D(\ell)=V \cdot \ell \tilde{V} \cdot \ell-\Delta_{\text {eff }}^{2}+i \epsilon$. We note that the contribution proportional to $J$ in (160) arises from the diagram of fig $2 \mathrm{~b}$ and corresponds to the lagrangian $\mathcal{L}_{2}$ in (1).

The integrals $I_{1}(p)$ and $\tilde{I}_{1}(p)$ are infrared divergent in the blocking region where $\Delta_{\text {eff }}=0$. One has to regularize them going to finite temperature and imaginary frequencies $\left(\ell_{0} \rightarrow 2 \pi i T(n+1 / 2)\right)$ (for a similar case see [20], eqns. 
(2.193) and (2.194)). The results that we get for the blocking and the pairing region are respectively:

$$
I_{1}(0)=I_{1}(p)=\tilde{I}_{1}(0)=\tilde{I}_{1}(p)=-2 \pi i, \quad I_{2}(0)=I_{2}(p)=0, \quad J=\frac{\pi i}{2} \mu^{2}
$$

and

$$
I_{1}(0)=I_{1}(p)=\tilde{I}_{1}(0)=\tilde{I}_{1}(p)=-\pi i, \quad I_{2}(0)=-\pi i, \quad J=\frac{\pi i}{2} \mu^{2} .
$$

From these we can easily compute the Debye and Meissner masses using

$$
\begin{aligned}
-\left.\Pi_{a b}^{\mu \nu}(0)\right|_{\text {blocking }} & =\delta_{a b} \frac{g^{2} \mu^{2}}{2 \pi^{2}} \sum_{\vec{v} ; \text { locking }}\left[2\left(V^{\mu} V^{\nu}+\tilde{V}^{\mu} \tilde{V}^{\nu}\right)\right. \\
& \left.+2 g^{\mu \nu}-\tilde{V}^{\mu} V^{\nu}-\tilde{V}^{\nu} V^{\mu}\right] \\
-\left.\Pi_{a b}^{\mu \nu}(0)\right|_{\text {pairing }} & =\delta_{a b} \frac{g^{2} \mu^{2}}{2 \pi^{2}} \sum_{\vec{v} ; \text { pairing }}\left[V^{\mu} V^{\nu}+\tilde{V}^{\mu} \tilde{V}^{\nu}\right. \\
& \left.+2 g^{\mu \nu}-2\left(\tilde{V}^{\mu} V^{\nu}+\tilde{V}^{\nu} V^{\mu}\right)\right] .
\end{aligned}
$$

We get therefore the vanishing of the Meissner mass

$$
\Pi_{a b}^{i j}(0)=0 .
$$

On the other hand we get a non-vanishing Debye screening mass from the blocking region:

$$
-\Pi_{a b}^{00}(0)=2 \delta_{a b} \frac{g^{2} \mu^{2}}{\pi^{2}} \sum_{\vec{v} ; \text { blocking }}=\delta_{a b} \frac{g^{2} \mu^{2}}{2 \pi^{2}} \int_{\text {blocking }} d \cos \theta,
$$

where

$$
\int_{\text {blocking }} d \cos \theta=\int_{-1}^{\cos \theta_{a}} d \cos \theta+\int_{\cos \theta_{b}}^{1} d \cos \theta
$$

The domain of integration of the blocking region has been obtained by the condition

$$
R|h(\cos \theta)|>\frac{\pi}{2}
$$

which gives, using the approximate expression for $h(\cos \theta)$ (30), and for $R>$ $\pi / 2$ :

$$
\cos \theta_{a}=\frac{\cos \theta_{q}}{1+\frac{\pi}{2 R}}, \quad \cos \theta_{b}=\min \left\{1, \frac{\cos \theta_{q}}{1-\frac{\pi}{2 R}}\right\}
$$


where $\cos \theta_{q}=\delta \mu / q$. From the equations (170) and (172) one gets the Debye mass:

$$
m_{D}=\frac{g \mu}{\pi} \sqrt{1+\frac{\cos \theta_{a}-\cos \theta_{b}}{2}} .
$$

These results can be easily generalized to the case of the cube.

\section{References}

[1] B. Barrois, Nucl. Phys. B129 (1977) 390; S. Frautschi, Proceedings of workshop on hadronic matter at extreme density, Erice 1978; D. Bailin and A. Love, Phys. Rept. 107 (1984) 325.

[2] M. Alford, K. Rajagopal and F. Wilczek, Phys. Lett. B422 (1998) 247, hep-ph/9711395; R. Rapp, T. Schäfer, E. V. Shuryak and M. Velkovsky, Phys. Rev. Lett. 81 (1998) 53, hep-ph/9711396.

[3] For a review see K. Rajagopal and F.Wilczek,in Handbook of QCD, M. Shifman ed. (World Scientific 2001), hep-ph/0011333.

[4] M. Alford, K. Rajagopal and F. Wilczek, Nucl. Phys. B537 (1999) 443, hep-ph/9804403.

[5] M. Alford, J. Bowers and K. Rajagopal, Phys. Rev. D63 (2001) 074016, hep-ph/0008208.

[6] J.A. Bowers, J. Kundu, K. Rajagopal and E. Shuster, Phys. Rev. D64 (2001) 014024, hep-ph/0101067.

[7] R. Casalbuoni, R. Gatto, M. Mannarelli and G. Nardulli, Phys. Lett. B 511 (2001) 218, hep-ph/0101326.

[8] A.K. Leibovich, K. Rajagopal and E. Shuster, Phys. Rev. D64 (2001) 094005, hep-ph/0104073; J. Kundu and K. Rajagopal, Phys. Rev. D65 (2002) 094022, hep-ph/0112206.

[9] For reviews see for instance: K. Rajagopal, in the Proceedings of QCD@Work: International Workshop on QCD: Theory and Experiment, Martina Franca, Bari, Italy (2001), editors P. Colangelo and G. Nardulli, AIP Conference Proceedings, V. 602 (2001), 
hep-ph/0109135; R. Casalbuoni, ibidem, hep-th/0108195; G. Nardulli, Proceedings of the Compact Stars in the QCD Phase Diagram, Copenhagen, Aug. 2001, eConf C010815:104-109,2002, eds. R. Ouyed and F. Sannino, hep-ph/0111178; M. Mannarelli, talk given at the Giselda Meeting, Frascati (Roma) (2001), hep-ph/0205098.

[10] A. I. Larkin and Yu. N. Ovchinnikov, Sov. Phys. JETP 20 (1965) 762; P. Fulde and R. A. Ferrell, Phys. Rev. 135 (1964) A550.

[11] J.A. Bowers and K. Rajagopal, hep-ph/0204079.

[12] G. Nardulli, talk given at the Giselda Meeting, Frascati (Roma) (2001), hep-ph/0206065.

[13] F. Schreck et al. Phys. Rev. Lett. 87, 080403 (2001), and references therein.

[14] M. Holland, S. J. J. M. F. Kokkelmans, M. L. Chiafolo, and R. Walser, Phys. Rev. Lett. 87, 120406 (2001), and references therein.

[15] R. Combescot, Europhys. Lett. 55, 150 (2001).

[16] N. Isgur and M.B. Wise, Phys. Lett. B232 (1989) 113 and Phys. Lett. B237 (1990) 527; E. Eichten and B. Hill, Phys. Lett. B234 (1990) 511; H. Georgi, Phys. Lett. B249 (1990) 447; for recent reviews see A. V. Manohar and M.B. Wise Heavy Quark Physics, Cambridge University Press (2000), and R. Casalbuoni, A. Deandrea, N. Di Bartolomeo, R. Gatto, F. Feruglio and G. Nardulli Phys. Rept.281 (1997) 145, hep-ph/9605342.

[17] D. K. Hong, Phys. Lett. B473 (2000) 118, hep-ph/9812510; D. K. Hong, Nucl. Phys. B582 (2000) 451, hep-ph/9905523.

[18] S.R. Beane, P.F. Bedaque and M.J. Savage, Phys. Lett. B483 (2000) 131, hep-ph/0002209.

[19] R. Casalbuoni, R. Gatto and G. Nardulli, Phys. Lett. B 498 (2001) 179 and Erratum-ibid. B517 (2001) 483, hep-ph/0010321.

[20] G. Nardulli, to appear in Rivista del Nuovo Cimento, hep-ph/0202037. 
[21] R. Casalbuoni, R. Gatto, M. Mannarelli and G. Nardulli, Phys. Rev. D66 (2002) 014006, hep-ph/0201059.

[22] See, for instance, M. Hamermesh, Group Theory and its application to Physical Problems, Addison Wesley, 1964.

[23] R. Casalbuoni, R. Gatto and G. Nardulli, hep-ph/0205219.

[24] L.D. Landau and E. M. Lifshitz, Course of Theoretical Physics, Vol. 7, Elasticity Theory, Pergamon, London, 1959.

[25] D.H. Rischke, D.T. Son and M.A. Stephanov, Phys. Rev. Lett. 87 (2001) 062001, hep-ph/0011379.

[26] R. Casalbuoni, R. Gatto, M. Mannarelli and G. Nardulli, Phys.Lett. B 524 (2002) 144, hep-ph/0107024.

[27] D. H. Rischke, Phys. Rev. D 62 (2000) 034007 , nucl-th/0001040. 